\title{
Caracterização agronômica e edafoclimática dos vinhedos de elevada altitude
}

\author{
Agronomic and edaphoclimatic characterization high altitude vineyards
}

\section{Luiz Fernando Vianna*, Angelo Mendes Massignan, Cristina Pandolfo, Denilson Dortzbach e Valci Francisco Vieira}

Recebido em 11/06/2015 / Aceito em 02/08/2016

\section{RESUMO}

O objetivo desse trabalho foi realizar a caracterização agronômica e edafoclimática dos vinhedos e determinar as tendências de uso das principais variedades para produzir vinhos finos nas regiões de elevada altitude do Estado de Santa Catarina. Foram considerados vinhedos de elevada altitude os vinhedos localizados acima de $900 \mathrm{~m}$ em relação ao nível do mar e os vinhedos pertencentes aos associados da Associação Catarinense de Vinhos Finos de Altitude (Acavitis) localizados em altitudes inferiores a 900 m. Para avaliar as tendências de cultivo foi realizado um cadastro georreferenciado, seguindo as normas da Embrapa Uva e Vinho. Para realizar a caracterização edafoclimática, as áreas dos vinhedos foram cruzadas com mapas fisiográficos, climáticos e pedológicos em um Sistema de Informações Geográficas. A caracterização dos vinhedos de elevada altitude foi realizada com base na área plantada. As variedades com maior área foram Cabernet Sauvignon (37,9\%), Merlot (14,5\%), Chardonnay (8,0\%) e Sauvignon Blanc (7,5\%). De 2009 a 2013 houve substituição de C. Sauvignon por variedades brancas e Pinot Noir, principalmente nas altitudes superiores a $1.100 \mathrm{~m}$. Houve uma tendência no uso de variedades brancas e Pinot Noir em vinhedos novos, mas a principal tendência observada foi no crescimento de áreas experimentais, com outras 60 variedades tintas e brancas. Em relação à fisiografia, a norma da Acavitis exige que os vinhedos dos associados estejam localizados acima de $900 \mathrm{~m}$ de altitude, porém foram encontrados vinhedos em altitudes entre de $675 \mathrm{~m} \mathrm{e}$ $1.452 \mathrm{~m}$, com $64,6 \%$ da área plantada entre $1.100 \mathrm{e}$ $1.300 \mathrm{~m}$ acima do nível do mar. Os vinhedos estão em áreas onde a pluviosidade anual chegou a ser $240 \%$ superior aos valores considerados ótimos pela literatura especializada. A temperatura média anual variou entre $12^{\circ} \mathrm{C}$ e $18{ }^{\circ} \mathrm{C}$ e tanto a amplitude térmica quanto as horas anuais de sol foram consideradas suficientes para completar o ciclo de todas as variedades.

PALAVRAS-CHAVE: cadastro vitícola, vinhos de altitude, vitivinicultura, vinhos brasileiros.

\section{ABSTRACT}

The objective of this research was to perform the agronomic and edaphoclimatic characterization of vineyards and to determine cultivation trends for the main varieties employed in the production of high altitude fine wines in Santa Catarina State, Brazil. High altitude vineyards were considered those vineyards located $900 \mathrm{~m}$ above sea level and vineyards belonging to members of the Santa Catarina Association of Altitude Fine Wines located below $900 \mathrm{~m}$ altitude. To assess cultivation trends, the vineyards were geo-referenced, following the Embrapa Uva e Vinho guidelines. To perform the edaphoclimatic characterization, the vineyard polygons were overlaid with physiographic, climatic and soil maps in a Geographic Information System. The characterization of the high altitude vineyards was based on planted area. The varieties with greatest areas were Cabernet Sauvignon (37.9\%), Merlot (14.5\%) Chardonnay (8.0\%) and Sauvignon Blanc (7.5\%). From 2009 to 2013 vineyards with C. Sauvignon were replaced for white varieties and for Pinot Noir, especially in altitudes over $1,100 \mathrm{~m}$. A trend in the use of white varieties and Pinot Noir in new vineyards was identified, but the main trend was the increase of experimental areas, with additional 60 varieties of red and white grapes. In relation to

Empresa de Pesquisa Agropecuária e Extensão Rural de Santa Catarina, Florianópolis, SC, Brasil.

*Autor para correspondência <vianna@epagri.sc.gov.br> 
physiography, the norm of the Acavitis requires that the vineyards of their associates be located above $900 \mathrm{~m}$ of altitude. However, vineyards were found at altitudes ranging from $675 \mathrm{~m}$ to $1,452 \mathrm{~m}$, with $64.6 \%$ of the area found between altitudes ranging from 1,100 to $1,300 \mathrm{~m}$ above sea level. The annual rainfall was $240 \%$ higher than the values considered optimal described in the literature for high altitude vineyards. The average annual temperature ranged between $12{ }^{\circ} \mathrm{C}$ and $18{ }^{\circ} \mathrm{C}$ and both the temperature range as the annual hours of sunshine were considered sufficient to complete the cycle of all varieties.

KEYWORDS: vineyard geo-referencing, high altitude wines, viticulture, Brazilian wines.

\section{INTRODUÇÃO}

A vitivinicultura das regiões de elevada altitude de Santa Catarina é recente (BRIGHENTI et al. 2013) porém já se destaca pela qualidade dos vinhos elaborados (FALCÃO et al. 2007, BURIN et al. 2011, GRIS et al. 2011). A qualidade dos vinhos de altitude está associada às características geográficas dessas regiões (MALINOVSKI et al. 2012, BORGHEZAN et al. 2014), aliada ao desenvolvimento de pesquisas focadas nos processos agronômicos, enológicos e fenológicos (FALCÃO et al. 2007, BORGHEZAN et al. 2011, BRIGHENTI et al. 2013), nas características climáticas (MALINOVSKI et al. 2012) e no potencial varietal (BRIGHENTI et al. 2014).

No entanto, para que se façam estudos integrados que abranjam aspectos ambientais, varietais e de tecnologia de produção, a caracterização agronômica e edafoclimática de uma região produtora de uvas para vinhos finos é um dos componentes necessários ao conhecimento da sua variabilidade produtiva e ambiental. O melhor instrumento para isso, segundo a Embrapa Uva e Vinho, é o cadastro vitícola (FIALHO et al. 2005, MELLO \& MACHADO 2013), que fornece informações importantes para a gestão da produção e da pesquisa científica.

Sabe-se que existem fatores ambientais, como temperatura, amplitude térmica, pluviosidade e composição físico-química do solo, que possuem correlação com componentes químicos, indicadores de qualidade do vinho (KLIEWER 1970, KLIEWER \& TORRES 1972, REYNOLDS et al. 1986, JACKSON \& LOMBARD 1993, SPAYD et al. 2002, FALCÃO et al. 2007, VAN LEEUWEN 2010, CHAVARRIA et al.
2011). Porém, segundo VAUDOUR (2002), JONES et al. (2004) e SANTOS (2006) a qualidade da uva e do vinho não está relacionada apenas às características geradas pela interação entre fatores edafoclimáticos, mas também com as práticas de cultivo. Esses autores são unânimes em destacar a complexidade que existe na identificação da influência de cada fator nessa qualidade.

Desta forma, o levantamento de dados agroclimáticos, pedológicos e fisiográficos numa escala que permita caracterizar, nos vinhedos, a variabilidade de processos agronômicos e ambientes é de suma importância para entender as interações no ecossistema vitícola. Com isto, o objetivo desse trabalho foi realizar a caracterização agronômica e edafoclimática dos vinhedos de elevada altitude do Estado de Santa Catarina e determinar as tendências de uso das principais variedades para elaboração de vinhos de altitude.

\section{MATERIAL E MÉTODOS}

Segundo a Associação Catarinense de Produtores de Vinhos Finos de Altitude - Acavitis (PROTAS 2012), consideram-se vinhedos de elevada altitude aqueles localizados acima de $900 \mathrm{~m} \mathrm{em}$ relação ao nível do mar. Apesar dessa definição, alguns vinhedos de associados da Acavitis encontramse abaixo de $900 \mathrm{~m}$. Nesse estudo foram considerados como vinhedos de elevada altitude para produção de vinhos finos: (i) os vinhedos de uvas finas localizados em altitudes superiores a $900 \mathrm{~m}$ e (ii) os vinhedos localizados abaixo de $900 \mathrm{~m}$ de altitude dos associados a Acavitis (Figura 1).

O cadastro vitícola foi feito seguindo as exigências cartográficas da Lei 10.267 (BRASIL 2001) e os conceitos e normas para cadastro vitícola da Embrapa Uva e Vinho (FIALHO et al. 2005). O detalhamento do método de georreferenciamento dos vinhedos de elevada altitude nesse cadastro foi publicado por VIEIRA \& ZAMPARETTI (2010). Todas as estatísticas e cálculos de área foram feitos com base nos setores (FIALHO et al. 2005), porém, para facilitar a interpretação dos resultados e o entendimento dos leitores, adotou-se o termo vinhedo como sinônimo de setor. Assim, para este artigo, entende-se como vinhedo uma área contínua plantada com uva de uma mesma variedade, porta-enxerto, sistema de condução e espaçamento. 


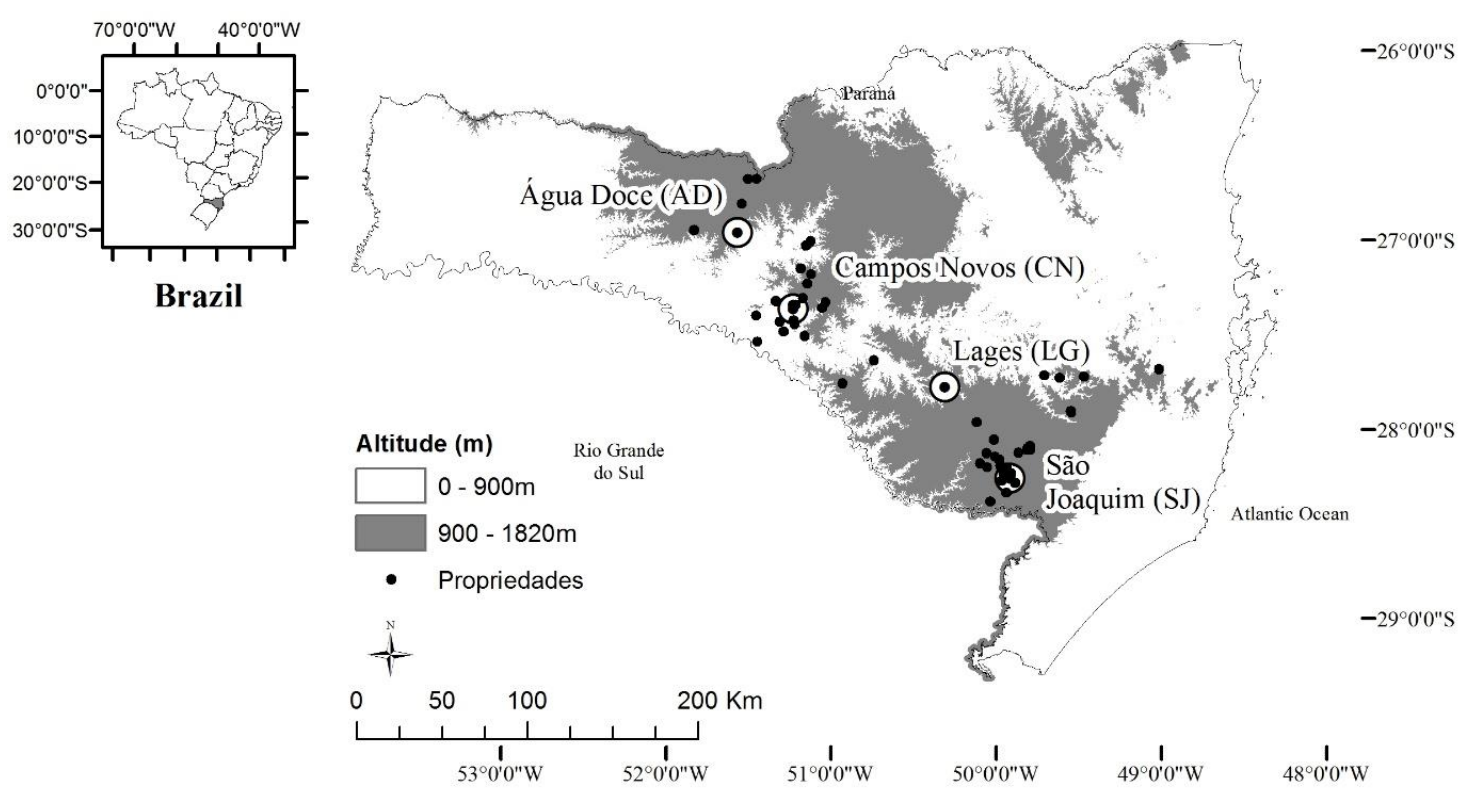

Figura 1 - Localização geográfica do estado de Santa Catarina no Brasil, delimitação das regiões de elevada altitude (900-1.820 m) e distribuição espacial das 53 propriedades produtoras de uvas para vinhos finos em 2013 no estado de Santa Catarina.

Figure 1 - Geographical location of Santa Catarina state in Brazil, delimitation of high altitude regions (900$1,820 \mathrm{~m}$ ) and spatial distribution of the 53 properties producing fine wine grapes in 2013 in the state of Santa Catarina.

O cadastro dos vinhedos de elevada altitude foi feito em duas etapas: a primeira foi realizada de junho de 2008 a novembro de 2009 e a segunda, de fevereiro a agosto de 2013. Para cada vinhedo foram cadastrados o ano de plantio, a variedade, o portaenxerto, o sistema de condução, o espaçamento entre filas, o espaçamento entre plantas e a área em hectares. Os dados foram organizados em um banco de dados georreferenciados no Sistema de Informações Geográficas ArcGis, versão 9.3 e depois atualizado para a versão 10.1.

Através da ferramenta de análise espacial estatística por zonas, os dados fisiográficos, climáticos e as classes de solos foram extraídos de mapas específicos para os polígonos dos vinhedos de elevada altitude. Foram utilizados mapas gerados a partir dos modelos digitais de elevação da Shuttle Radar Topographic Mission - SRTM (FARR et al. 2007) e da Secretaria Estadual de Desenvolvimento Sustentável - SDS (SDS 2013), com base nos modelos de regressão para estimativa de temperaturas médias anuais de MASSIGNAN \& PANDOLFO (2006). Também foram usados mapas de estimativa de horas de sol e mapas de pluviosidade do Atlas Climatológico de Santa Catarina (PANDOLFO et al. 2007). Além do mapa de solos 1:250.000 do estado de Santa Catarina (EMBRAPA 2004). Com isso foram calculados, para cada vinhedo, a altitude, a declividade, a orientação, as temperaturas médias, a média do total de horas de sol anuais, a pluviosidade média anual, a amplitude térmica diária média e as classes de solo.

As estatísticas descritivas foram calculadas no ArcGis 10.1 e os gráficos gerados no Excel.

\section{RESULTADOS E DISCUSSÃO}

\section{Histórico de plantio dos vinhedos e principais variedades cultivadas}

Os primeiros vinhedos de elevada altitude em Santa Catarina para elaboração de vinhos finos foram plantados ao final dos anos 90 . Até 2013, 590 vinhedos tinham sido implantados, totalizando uma área de 332,35 ha distribuídas em 53 propriedades. A maior expansão em plantio ocorreu entre 2002 e 2006, com destaque o ano de 2005, quando foram plantadas quase $18 \%$ da área atual (Figura 2).

A primeira década de implantação dos vinhedos de elevada altitude apresentou uma taxa média de 
plantio de 25,8 ha $a n o^{-1}$. A partir de 2008 a taxa de plantio reduziu-se para 17,6 ha $\mathrm{ano}^{-1}$, mas mesmo assim a atividade continuou expandindo.

Os vinhedos de elevada altitude estão distribuídos em 13 municípios (Tabela 1). Entre 2009 e 2013 houve um acréscimo de 73,6 ha de área plantada (28,4\% em relação a 2009). Esse acréscimo ocorreu com maior representatividade nos municípios de São Joaquim (14,1\%), Bom Retiro $(6,1 \%)$ e Água Doce (3,1\%). Nos municípios de Painel, Rancho Queimado e Vargem Bonita, não houve alteração de área entre 2009 e 2013. Já nos municípios de Campos Novos e Tangará, foi identificada uma redução de $0,3 \%$ em cada um, em relação à área de 2009.

Analisando apenas os dados de 2013, dos 332,35 ha de vinhedos, os municípios de São Joaquim e Água Doce concentraram a maior área, que corresponderam a 50,6\% e $15,5 \%$ da área total, respectivamente. As propriedades com as maiores áreas individuais estão em Água Doce, com média de 17,2 ha de vinhedos por propriedade, Urupema (16,7 ha/propriedade) e Tangará (11,1 ha/propriedade). São Joaquim foi o município com maior número de propriedades $(39,62 \%)$, com uma área média de 7,6 ha/propriedade.

De acordo com o regulamento da marca coletiva Acavitis, a produtividade máxima nos vinhedos de elevada altitude deve ser de $6.000 \mathrm{~L} \mathrm{ha}^{-1}$, o que confere a área levantada em 2013 um potencial produtivo máximo de 1.994.100 litros por safra. O regulamento também exige que os vinhos sejam produzidos apenas com uvas Vitis vinifera, que correspondem a 93,63\% da área plantada, sendo $72,58 \%$ tintas e $21,05 \%$ brancas. Foram identificadas 65 variedades cultivadas nos vinhedos de elevada altitude. Há alguns vinhedos com diferentes variedades, inclusive algumas americanas, associados normalmente a experimentos ou coleções particulares, que cobrem $6,37 \%$ da área cultivada.

Na Tabela 2 estão apresentados os dados de área total das variedades mais cultivadas em hectares e porcentagem nos anos de 2009 e 2013. Em 2009 nos vinhedos de elevada altitude, as variedades tintas Cabernet Sauvignon e Merlot foram as que apresentaram maior área plantada, seguindo uma tradição observado também no Rio Grande do Sul (MELLO \& MACHADO 2013). Enquanto para as variedades brancas, observou-se maior área plantada com Chardonnay e Sauvignon Blanc. Essas quatro variedades mantiveram-se como as mais representativas em área, em 2013, porém houve alteração na proporção em relação a área total.

A Cabernet Sauvignon ainda é a variedade com maior área plantada, mas em relação a área de 2009, foi observada uma redução de $1,1 \%$. Entre 2009 e 2013 foram plantados 11,27 ha de vinhedos com esta variedade, porém 12,66 ha já estabelecidos foram sobre enxertados ou eliminados. A maioria dos vinhedos de elevada altitude com Cabernet Sauvignon sobre enxertados ou eliminados $(90,9 \%)$ estavam em locais com altitudes superiores a $1.100 \mathrm{~m}$.

Outras 61 variedades estão sendo testadas, o que justifica o aumento significativo na área plantada com variedades tintas e brancas em pequenos vinhedos. Entre as variedades tintas que apresentaram uma tendência de aumento na área plantada de 2009 para 2013 estão a Sangiovese, que segundo BRIGHENTI et al. (2014), é uma das variedades tintas mais bem adaptadas às condições de altitude de Santa Catarina. A outra variedade é a Pinot Noir, que apesar de tinta, é muito utilizada para produção de espumantes e apresentou uma porcentagem de aumento de área em relação a 2009 próxima àquelas observadas para as variedades brancas Chardonnay e Sauvignon Blanc. Essas últimas não só tiveram suas áreas ampliadas em 2013 como aumentaram sua representatividade em relação a área total dos vinhedos.

Entre 2009 e 2013 a sobre enxertia foi responsável pela alteração de $3,8 \%$ da área cultivada. Esse processo indicou uma tendência pela preferência de algumas variedades e também pela substituição de uvas tintas por brancas. Nos vinhedos de elevada altitude sobre enxertados, entre 2009 e 2013, houve um acréscimo de 3,4ha para variedades brancas e uma redução de 6,55 ha para variedades tintas. Tanto a expansão de área plantada quanto a sobre enxertia de vinhedos de uvas tintas com uvas brancas e com Pinot Noir indicam uma tendência de aumento na elaboração de vinhos brancos e espumantes a partir de 2009.

Os dados do levantamento das variedades cultivadas nos vinhedos de elevada altitude, sua quantificação em termos de área plantada e as datas de plantio favoreceram a apresentação de um panorama que aponta tendências de produção para os vinhos finos de altitude. Isso justifica a importância da manutenção do cadastro vitícola de forma operacional e sistemática, para que seja possível acompanhar essa tendência, uma vez que se trata de uma atividade recente. 


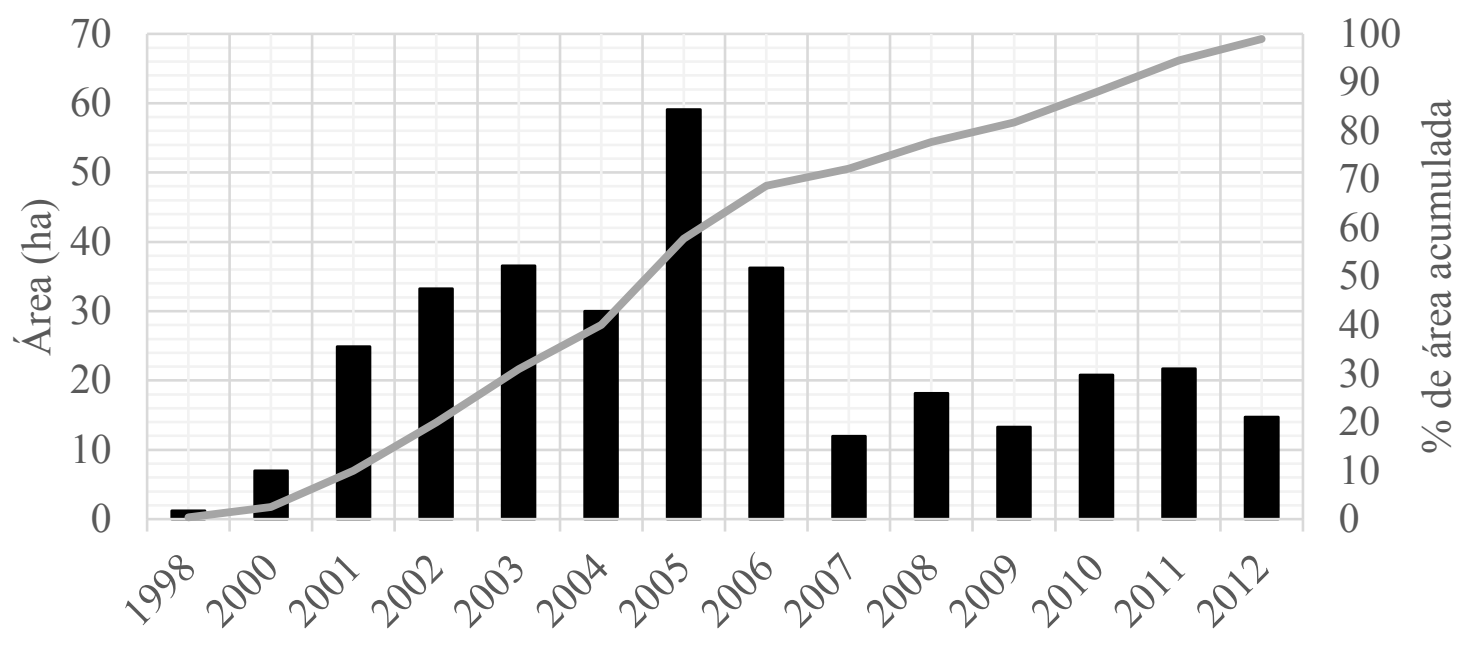

Ano de plantio

Figura 2 - Área (ha) dos vinhedos de elevada altitude implantados por ano de plantio e porcentagem de área acumulada no período de 1998 a 2012 no estado de Santa Catarina.

Figure 2 - Total area of high altitude vineyards by year and percentage of cumulative area from 1998 to 2012 in the state of Santa Catarina.

Tabela 1 - Número de propriedades, número e área total dos vinhedos de elevada altitude por município nos anos de 2009 e 2013 no estado de Santa Catarina.

Table 1 - Number of properties, number of high altitude vineyards and total area of the high altitude vineyards by municipality in 2009 and 2013 in the state of Santa Catarina.

\begin{tabular}{|c|c|c|c|c|c|c|}
\hline \multirow[t]{2}{*}{ Município } & \multicolumn{3}{|c|}{2009} & \multicolumn{3}{|c|}{2013} \\
\hline & Propriedades & Vinhedos & Área (ha) & Propriedades & Vinhedos & Área (ha) \\
\hline Água Doce & 2 & 62 & 43,55 & 3 & 79 & 51,59 \\
\hline Bom Retiro & 0 & 0 & 0,00 & 3 & 50 & 15,72 \\
\hline Campo Belo do Sul & 1 & 13 & 4,04 & 1 & 19 & 7,28 \\
\hline Campos Novos & 13 & 62 & 23,31 & 14 & 61 & 22,48 \\
\hline Cerro Negro & 0 & 0 & 0,00 & 1 & 5 & 2,35 \\
\hline Painel & 1 & 10 & 0,94 & 1 & 10 & 0,94 \\
\hline Rancho Queimado & 1 & 7 & 2,20 & 1 & 7 & 2,20 \\
\hline São Joaquim & 18 & 181 & 131,63 & 21 & 268 & 168,13 \\
\hline Tangará & 2 & 15 & 23,01 & 2 & 14 & 22,18 \\
\hline Urubici & 1 & 9 & 1,28 & 2 & 29 & 8,33 \\
\hline Urupema & 1 & 16 & 15,83 & 1 & 17 & 16,68 \\
\hline Vargem Bonita & 1 & 7 & 1,63 & 1 & 7 & 1,63 \\
\hline Videira & 2 & 24 & 11,31 & 2 & 24 & 12,84 \\
\hline TOTAL & 43 & 406 & 258,74 & 53 & 590 & 332,35 \\
\hline
\end{tabular}


Tabela 2 - Área total e porcentagem de área dos vinhedos de elevada altitude nos anos de 2009 e 2013 e porcentagem de aumento de área em relação a área plantada de 2009 por variedade no estado de Santa Catarina.

Table 2 - Total area and percent of area of high altitude vineyards in 2009 and 2013 and percent of increasing area from 2009 by grape variety in the state of Santa Catarina.

\begin{tabular}{lrrrrc}
\hline Variedade & \multicolumn{2}{c}{2009} & \multicolumn{2}{c}{2013} & $2009-2013$ \\
\cline { 2 - 6 } & Área (ha) & $\%$ de área & Área (ha) & $\%$ de área & $\begin{array}{c}\% \text { de aumento em } \\
\text { relação a 2009 }\end{array}$ \\
& & & & & $-1,1 \%$ \\
Cabernet Sauvignon & 127,28 & $49,2 \%$ & 125,89 & $37,9 \%$ & $8,5 \%$ \\
Merlot & 44,48 & $17,2 \%$ & 48,25 & $14,5 \%$ & $70,1 \%$ \\
Chardonnay & 15,72 & $6,1 \%$ & 26,75 & $8,0 \%$ & $68,9 \%$ \\
Pinot Noir & 14,70 & $5,7 \%$ & 24,83 & $7,5 \%$ & $67,1 \%$ \\
Várias & 10,40 & $4,0 \%$ & 17,37 & $5,2 \%$ & $37,7 \%$ \\
Sangiovese & 10,84 & $4,2 \%$ & 14,93 & $4,5 \%$ & $331,7 \%$ \\
Malbec & 2,60 & $1,0 \%$ & 11,20 & $3,4 \%$ & $32,8 \%$ \\
Cabernet Franc & 7,14 & $2,8 \%$ & 9,49 & $2,9 \%$ & $9,6 \%$ \\
Outros & 5,96 & $2,3 \%$ & 6,54 & $2,0 \%$ & $140,1 \%$ \\
Total & 19,62 & $7,6 \%$ & 47,10 & $14,2 \%$ & $28,4 \%$ \\
\hline
\end{tabular}

\section{Caracterização agronômica}

Os proprietários dos vinhedos de elevada altitude procuram seguir um padrão de espaçamento entre plantas e filas de $1,5 \mathrm{~m} \times 3,0 \mathrm{~m}$, com algumas variações, conforme recomendado pela Embrapa Uva e Vinho. Em média, considerando o total de área plantada em 2013, o espaçamento entre plantas foi de 1,33 m (mínimo 0,7; máximo 3,0 m), e entre filas, de 2,96 m (mínimo 1,2 m; máximo 4,0 m). De acordo com a Embrapa Uva e Vinho, o espaçamento ideal pode variar de acordo com a topografia do terreno e o sistema de condução.

O sistema de condução mais usado nos vinhedos foi a espaldeira $(84,76 \%$ da área). Trata-se de uma orientação da marca coletiva Acavitis para reduzir a produtividade. Contudo, outros sistemas também foram identificados: Manjedoura esteve presente em $10,17 \%$ da área, Latada em 1,71\%, Lira em $0,11 \%$ e $3,26 \%$ da área dos vinhedos apresentaram sistemas de condução diversos.

A escolha do sistema de condução e as práticas de manejo favorecem o controle das condições microclimáticas nos vinhedos. O maior controle das condições microclimáticas auxilia na relação crescimento vegetativo-produção e na elevação do potencial enológico da uva (SANTOS 2006). A escolha do sistema de condução deve ser feita de acordo com as características fisiográficas do local de implantação do vinhedo.

Outro aspecto agronômico importante é a definição do porta-enxerto. O Paulsen 1103 foi o porta-enxerto predominante nos vinhedos de elevada altitude, presente em $72,1 \%$ da área plantada até 2013. Sua predominância se deve ao fato de ser o porta-enxerto que apresenta menor produtividade (BRIGHENTI et al. 2011) e resistência à fusariose, sendo utilizado desde os anos 90, predominantemente, no Sul do Brasil (CAMARGO et al. 2011). Os portaenxertos 4343 e SO4 foram encontrados em 5,8\% e $4,4 \%$ da área dos vinhedos. Em 4,2\% da área os portaenxertos não foram identificados e os $13,5 \%$ da área dos vinhedos restantes, corresponderam a pequenos vinhedos com outros 21 porta-enxertos.

Os dados relacionados aos aspectos agronômicos demonstraram que existe uniformidade na implantação dos vinhedos. Tanto os espaçamentos quanto o sistema de condução e a escolha do portaenxerto, seguiram padrões sugeridos pela Embrapa e pelas normas da Acavitis. 


\section{Caracterização edafoclimática}

Na Figura 3 estão apresentados os resultados das estatísticas da área dos vinhedos de elevada altitude por classes de altitude, declividade, orientação, temperatura média anual, amplitude térmica média diária, pluviosidade média anual, média do total de horas de sol anuais e solos.

A altitude média dos vinhedos de elevada altitude cadastrados até 2013 foi de $1.136 \mathrm{~m}$, o mais baixo localizado a $675 \mathrm{~m}$ e o mais alto a $1.452 \mathrm{~m}$. A faixa de altitude entre 1.100 e $1.300 \mathrm{~m}$ foi a que apresentou a maior área plantada (64,6\%) (Figura $3 \mathrm{~A})$. Foi nessa faixa que também ocorreu a maior expansão de área entre 2009 e 2013 (12,4\%). Apesar do regulamento da marca coletiva Acavitis limitar a faixa mínima de altitude para seus vinhedos em $900 \mathrm{~m}$, foram identificados alguns vinhedos de associados em altitudes inferiores $(10,1 \%$ da área plantada).

A altitude vem sendo considerada como o principal diferencial para elaboração de vinhos finos em Santa Catarina (ROSIER 2006, FALCÃO et al. 2007, BORGHEZAN et al. 2011, BRIGHENTI et al. 2013, BRIGHENTI et al. 2014). Sua relação inversa com a temperatura faz com que o ciclo vegetativo das uvas seja mais longo, proporcionando um diferencial físico-químico e sensorial aos vinhos (ROSIER 2006, REGINA et al. 2010, BRIGHENTI et al. 2013).

Os vinhedos de elevada altitude apresentaram diferentes aspectos fisiográficos, entre eles uma variação na declividade (Figura 3B). A maior parte dos vinhedos de elevada altitude está em terreno classificado como suave ondulado, com declividades que variam entre 3 e $8 \%(39,4 \%)$, e ondulado, de 8 a $20 \%(37,2 \%)$, segundo o sistema de classificação proposto por UBERTI et al. (1992). A escolha de terrenos suavemente ondulados ou ondulados pode ser favorável para otimizar a insolação, que também depende da orientação dos vinhedos e do sistema de condução. Já a escolha de terrenos planos, com declividades inferiores a $3 \%(23,0 \%)$, facilita a mecanização.

A Figura 3C ilustra a distribuição da área dos vinhedos de elevada altitude de acordo com a orientação de plantio. Os vinhedos de elevada altitude encontram-se instalados preferencialmente no quadrante norte (N, NE e NO), com 194,1 ha (58,4\%). No quadrante oeste $(\mathrm{O}, \mathrm{SO})$ tem 75,5 ha $(22,7 \%)$ e no quadrante leste (E, SE) 45,4 ha (13,6\%). Voltados para o sul, apenas 17,3 ha $(5,2 \%)$.
Na vitivinicultura a orientação é uma variável considerada importante, principalmente nas regiões montanhosas (WOLF \& BOYER 2003, KURTURAL et al. 2006). Em São Joaquim, CAMPOS et al. (2013) quantificaram a partição de radiação solar em vinhedos de elevada altitude nas orientações leste, oeste e no topo do dossel. O trabalho foi feito em cultivos conduzidos em espaldeira com vinhedos de elevada altitude orientados no sentido norte-sul. Apesar de não identificarem a orientação fisiográfica do terreno, os autores concluíram que cerca de 30 a $40 \%$ da radiação solar global incidente no dossel está disponível nas faces leste e oeste, com valor superior para a face leste. Concluíram ainda que o plantio da videira no sentido norte-sul proporciona o recebimento de radiação solar durante todo o período de insolação.

Entre os vinhedos de elevada altitude foi observada uma variação na temperatura média anual de $12,9^{\circ} \mathrm{C}$ a $17,4{ }^{\circ} \mathrm{C}$ (Figura 3D). Em relação a 2009, o maior incremento de área ocorreu em locais com temperatura média anual de $13{ }^{\circ} \mathrm{C}$ a $14{ }^{\circ} \mathrm{C}(12,4 \%)$ seguido de locais com temperatura média anual de $15{ }^{\circ} \mathrm{C}$ a $16{ }^{\circ} \mathrm{C}(9,6 \%)$.

Entre os fatores climáticos, a temperatura é o principal diferencial das regiões de altitude (BRIGHENTI et al. 2013, ROSIER 2006, FALCÃO et al. 2007, BORGHEZAN et al. 2011, BRIGHENTI et al. 2014). BORGHEZAN et al. (2011) estudaram os estádios fenológicos das variedades Cabernet Sauvignon, Merlot e Sauvignon Blanc em São Joaquim e concluíram que todas apresentaram maturação tardia em relação a outras regiões do Brasil. Entre os vinhedos de elevada altitude que apresentaram temperatura média anual inferior a $15^{\circ} \mathrm{C}(59,0 \%$ da área total), estão os vinhedos situados em São Joaquim. A maturação tardia favorece uma maior concentração de açúcares que proporcionam corpo ao vinho. Além disso, as noites frias favorecem um incremento na coloração e nos níveis de acidez (KLIEWER \& TORRES 1972).

A amplitude térmica não diferiu muito entre as regiões de altitude de Santa Catarina (Figura 3E). A maioria dos vinhedos de elevada altitude apresentou amplitude térmica média diária entre $10{ }^{\circ} \mathrm{C}$ e $11{ }^{\circ} \mathrm{C}$ (79,0\% da área). Amplitude térmica é a diferença entre as temperaturas máxima e mínima diárias e influencia a concentração de compostos fenólicos que dão estrutura e cor aos vinhos (KLIEWER,1970, KLIEWER \& TORRES 1972). 

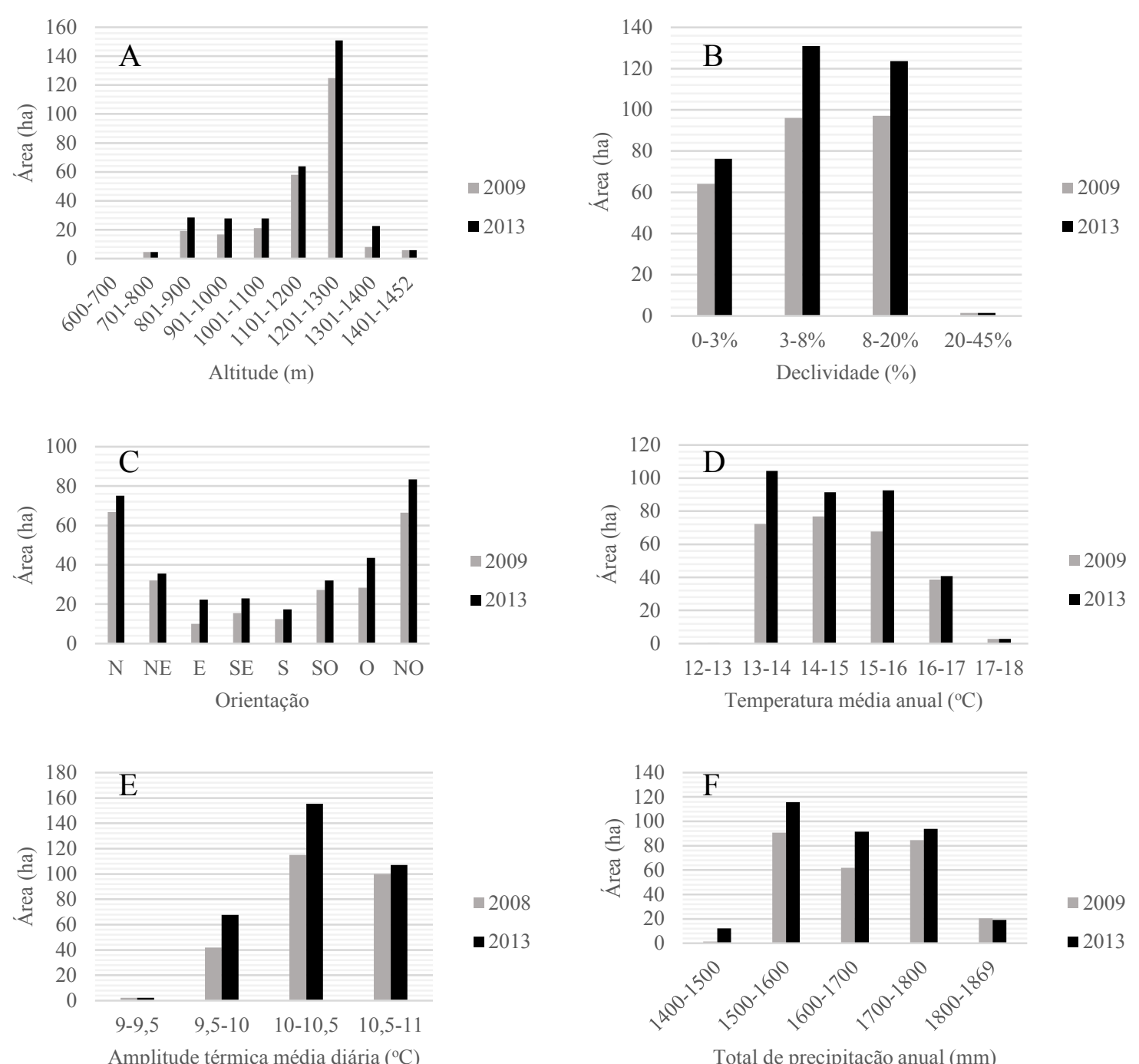

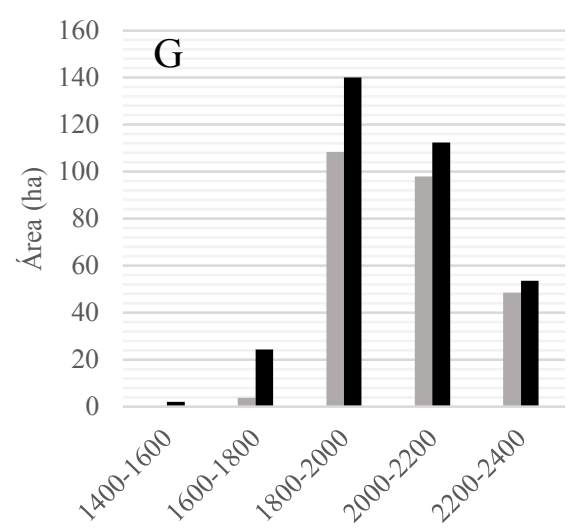

Total de horas de sol anuais

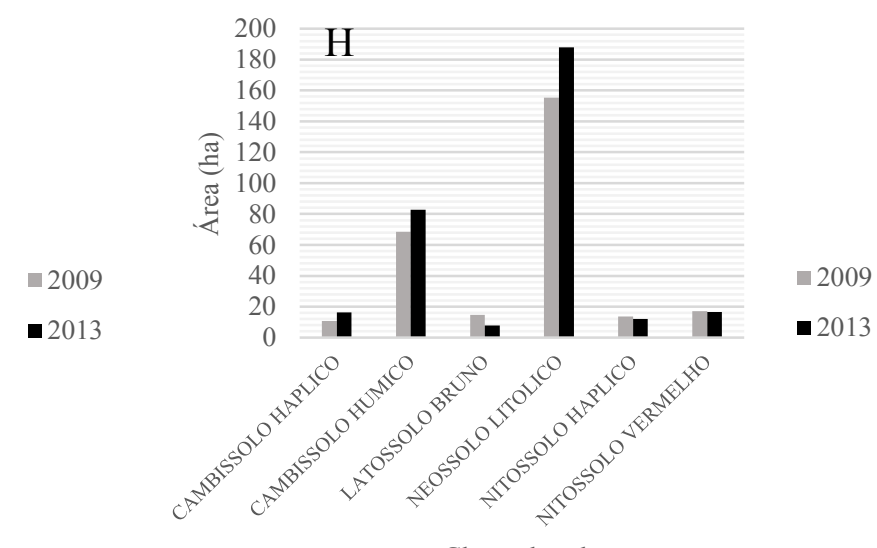

Figura 3 - Área total dos vinhedos de elevada altitude por faixa de altitude (A); declividade (B); orientação (C); temperatura média anual (D); amplitude térmica diária (E); total de precipitação anual (F); total de horas de sol anuais $(\mathrm{G})$ e classes de solos (H) nos anos de 2009 e 2013 no estado de Santa Catarina.

Figure 3 - Total area of high altitude vineyards by altitude (A); slope (B); aspect (C); average annual temperature $(D)$; daily temperature range $(E)$; total of annual precipitation $(F)$; annual sunlight hours $(G)$ and soil classes (H) in 2009 and 2013 in the state of Santa Catarina. 
GRIS et al. (2010) consideram $10{ }^{\circ} \mathrm{C}$ de amplitude térmica adequado para a fenologia $\mathrm{e}$ a maturação das uvas nas regiões de altitude de Santa Catarina. FALCÃO et al. (2010) avaliaram a influência da altitude e de fatores mesoclimáticos na fenologia e maturação da Cabernet Sauvignon nessas regiões, e perceberam que a amplitude térmica é mais significativa nos vinhedos de elevada altitude localizados em vales, do que naqueles localizados em planaltos. Esses autores concluíram ainda que a quantidade de noites frias é mais importante do que a amplitude térmica para a qualidade do vinho.

Em termos anuais, a pluviosidade média nos vinhedos de elevada altitude de Santa Catarina variou entre $1.400 \mathrm{~mm}$ e $1.869 \mathrm{~mm}$ (Figura 3F). A maior parte dos vinhedos de elevada altitude $(90,61 \%)$ situa-se em áreas com pluviosidade anual de 1.500 $\mathrm{mm}$ a $1.800 \mathrm{~mm}$. Esses índices são considerados altos em comparação aos 300 a $1.000 \mathrm{~mm}$ anuais das melhores regiões produtoras de vinho do mundo (VAN LEEUWEN 2010). Nas condições climáticas da Região Sul do Brasil, a fase de vegetação e produção do ciclo vegetativo das videiras coincide com o período de primavera e verão, quente e chuvoso (REGINA et al. 2006, REGINA et al. 2006b). Além da incidência de doenças fúngicas (SCOTT et al. 2010), essas condições induzem a um crescimento vegetativo vigoroso que influencia diretamente a maturação fenólica das uvas, impedindo que ela ocorra de forma completa (CHAMPAGNOL 1984).

O período mais importante para a qualidade da uva para vinho fino é aquele que compreende a maturação e a colheita (MANDELLI 2006). Segundo o autor, além dos dias ensolarados e com reduzida precipitação, a quantidade, a intensidade e a distribuição das precipitações são fundamentais para o equilíbrio na relação açúcar/acidez. Nesse sentido, ROSIER (2006), FALCÃO et al. (2008) e BORGHEZAN et al. (2014) afirmam que, em função das baixas temperaturas, o ciclo vegetativo nas regiões de altitude de Santa Catarina é mais longo, o que faz com que a colheita das variedades precoces ocorra a partir da segunda quinzena de março e a colheita das variedades tardias estenda-se pelo mês de abril, coincidindo com períodos de menor precipitação pluviométrica em comparação aos meses de verão e em comparação ao período de colheita em outras regiões tradicionais de produção de vinho no Brasil (Serra Gaúcha, RS e Vale do rio de Peixe, SC).

A distribuição da área plantada por horas de sol anuais está representada na Figura 3G. Entre os vinhedos de elevada altitude com menos horas de sol e aqueles com mais horas de sol há uma diferença de até 1.000 horas anuais. A maioria dos vinhedos de elevada altitude $(75,94 \%)$ recebe anualmente entre 1.800 e 2.200 horas de sol. Os mais ensolarados $(16,11 \%)$ recebem até 2.424 horas de sol anualmente.

O total de horas de sol anuais está diretamente associado as atividades fotossintéticas e metabólicas das videiras e, em relação a qualidade das uvas para o vinho, a insolação tem uma relação direta com a temperatura. Para variedades tintas o sombreamento dos cachos reduz a cor, a concentração de antocianina e os sólidos solúveis, aumentandoa acidez(REYNOLDS et al. 1986, SMART et al. 1988, FRANCESCO et al. 1994). Como a insolação está diretamente relacionada à orientação dos vinhedos, SPAYD et al. (2002) estudaram a influência da insolação na qualidade das uvas e comprovaram a existência de diferenças significativas nas concentrações de antocianinas em função da orientação dos vinhedos e do sombreamento dos cachos. SENTELHAS (1998) sugere que a videira necessita entre 1.200 e 1.400 horas de sol anuais para completar seu ciclo, dependendo da variedade.

Além da fisiografia e do clima, o solo também é um elemento importante na vitivinicultura (SARMENTO et al. 2008, CHAVARRIA et al. 2011). As características do solo influenciam diretamente sobre a composição da uva e do vinho e são dependentes do material de origem bem como dos demais fatores de formação: clima, relevo, organismos e tempo (VAN LEEUWEN \& SEGUIN 2006). Com base nas classes de solo do mapa de Santa Catarina 1:250.000 (EMBRAPA 2004), observa-se que mais da metade da área plantada com vinhedos de elevada altitude encontra-se na classe de Neossolo Litólico (56,53\%) e outros 24,94\% em Cambissolo Húmico (Figura 3H). Isso demonstra que, segundo o Sistema Brasileiro de Classificação de Solos - SiBCS (EMBRAPA 2013), os vinhedos de elevada altitude estão localizados em solos jovens, que apresentam predomínio das características herdadas do material geológico de origem. São solos pouco profundos, pedregosos e sem a presença de horizonte e diagnóstico, associados geralmente a relevos mais acidentados. Por serem pouco evoluídos, apresentam baixa fertilidade natural, necessitando de fertilização e correção de acidez antes da implantação dos vinhedos.

Em contrapartida os vinhedos de elevada altitude localizados sobre relevos menos acidentados, 
apresentaram Latossolo Bruno (2,4\% da área plantada). São solos profundos e muito porosos em condições naturais, possuem baixo potencial nutricional e altos teores de alumínio em profundidade, os quais podem afetar o enraizamento. Além disso, os elevados teores de argila associados aos elevados índices de pluviosidade das regiões de altitude não são os ideais para o cultivo da videira. Entre 2009 e 2013 Latossolo Bruno foi a classe de solo que apresentou a maior perda de área cultivada (6,75 ha) (Figura 3H).

A análise dos dados edafoclimáticos permitiu descrever um cenário ambiental que diferencia os vinhedos de elevada altitude de Santa Catarina dos vinhedos plantados em outras regiões do Brasil. Apresentou os pontos positivos e negativos do relevo, do clima e do solo e suas relações com o cultivo da videira para produção de vinhos finos. O cruzamento dos dados edafoclimáticos com o cadastro das variáveis e ano de plantio dos vinhedos favoreceu a identificação de uma tendência de aumento na produção uvas para vinhos brancos e espumantes e na produção da variedade tinta Sangiovese. Além disso permitiu identificar uma tendência de redução das áreas de Cabernet Sauvignon acima de 1.100 m de altitude.

\section{CONCLUSÃO}

Em termos agronômicos constatou-se a existência de um padrão no uso do sistema de condução em espaldeira, da adoção de porta-enxertos de baixa produtividade e resistência à fusariose e na estruturação dos vinhedos de elevada altitude em relação aos espaçamentos entre plantas e filas.

Os vinhedos estão em áreas onde a pluviosidade anual chegou a ser $240 \%$ superior aos valores considerados ótimos pela literatura especializada. A temperatura média anual variou entre $12{ }^{\circ} \mathrm{C}$ e $18{ }^{\circ} \mathrm{C}$ e tanto a amplitude térmica quanto as horas anuais de sol foram consideradas suficientes para completar o ciclo de todas as variedades.

Predominaram solos pouco evoluídos, associados a relevos acidentados, que precisam de análises prévias para avaliar a necessidade de fertilização e correção de $\mathrm{pH}$ para produção da videira.

A partir de 2009, foi identificada uma tendência de estagnação na ampliação das áreas plantadas com Cabernet Sauvignon e Merlot e uma tendência de ampliação das áreas com variedades brancas, Pinot Noir e Sangiovese. Alguns vinhedos com Cabernet
Sauvignon localizados acima de $1.100 \mathrm{~m}$ de altitude foram sobre-enxertados ou eliminados.

\section{REFERÊNCIAS}

BORGHEZAN M et al. 2011. Comportamento vegetativo e produtivo da videira e composição da uva em São Joaquim, Santa Catarina. Pesquisa Agropecuária Brasileira 46:398405.

BORGHEZAN M et al. 2014. Phenology and Vegetative Growth in a New Production Region of Grapevines: Case Study in São Joaquim, Santa Catarina, Southern Brazil. Open Journal of Ecology. 4:321-335.

BRASIL. 2001. Lei $\mathrm{n}^{\circ} 10.267$, de 28 de agosto de 2001. Dispõe sobre o georreferenciamento dos imóveis rurais. Disponível em: http://www.planalto.gov.br/ccivil_03/leis/ leis_2001/110267.htm Acesso em: 29 set. 2015.

BRIGHENTI AF et al. 2011. Desempenho vitivinícola da Cabernet Sauvignon sobre diferentes porta-enxertos em região de altitude de Santa Catarina. Revista Brasileira de Fruticultura 33:96-102.

BRIGHENTI AF et al. 2013. Caracterização fenológica e exigência térmica de diferentes variedades de uvas viníferas em São Joaquim, Santa Catarina - Brasil. Ciência Rural 43:1162-1167.

BRIGHENTI AF et al. 2014. Desempenho vitícola de variedades autóctones italianas em condição de elevada altitude no Sul do Brasil. Pesquisa Agropecuária Brasileira 49:465-474.

BURIN VM et al. 2011. Characterization and multivariate classification of grapes and wines of two Cabernet Sauvignon clones. Pesquisa Agropecuária Brasileira 46:474-481.

CAMARGO UA et al. 2011. Progressos na viticultura brasileira. Revista Brasileira de Fruticultura 33:144-149.

CAMPOS CGC et al. 2013. Fluxos de Radiação Solar Global em Vinhedos de Altitude de São Joaquim-SC. Revista Brasileira de Fruticultura 35:722-729.

CHAMPAGNOL F. 1984. Élements de physiologie de la vigne et de viticulture generale. Montpellier: DEHAN. $351 \mathrm{p}$.

CHAVARRIA G et al. 2011. Relações hídricas, rendimento e compostos fenólicos de uvas Cabernet Sauvignon em três tipos de solo. Bragantia 70:481-487.

EMBRAPA - EMPRESA BRASILEIRA DE PESQUISA AGROPECUÁRIA 2004. Solos do Estado de Santa Catarina (1:250.000). Rio de Janeiro: Embrapa Solos. 721p. (Boletim de Pesquisa e Desenvolvimento).

EMBRAPA - EMPRESA BRASILEIRA DE PESQUISA

AGROPECUÁRIA 2013. Sistema Brasileiro de Classificação de Solos. 3.ed. Brasília: Embrapa. 353p. FALCÃO LD et al. 2007. A survey of seasonal temperatures and vineyard altitude influences on 2-methoxy-3isobutylpyrazine, C13-norisprenoids, and the sensory profile of Brazilian Cabernet Sauvignon wines. Journal of 
Agricultural and Food Chemistry 55:3605-3612.

FALCÃO LD et al. 2008. Maturity of Cabernet Sauvignon Berries from Grapevines Grown with Two Different Training Systems in a New Grape Growing Region in Brazil. Ciencia e Investigación Agraria 35:271-282.

FALCÃO LD et al. 2010. Vineyard altitude and mesoclimate influences on the phenology and maturation of Cabernet Sauvignon grapes from Santa Catarina State. Journal International des Sciences de la Vigne et du Vin 44:135-150.

FARR TG et al. 2007. The Shuttle Radar Topography Mission, Reviews of Geophysics 45, RG2004.

FIALHOBFetal.2005.MetodologiadeGeorreferenciamento do Cadastro Vitícola. Bento Gonçalves: EMBRAPA-UVA E VINHO. 26p. (Documentos, 50).

FRANCESCO I et al. 1994. Differential effects of canopy manipulation and shading of Vitis vinifera L. cv. Cabernet Sauvignon. I. Composition of grape berries. Vitic. Enol. Sci. 49:220-225.

GRIS EF et al. 2010. Phenology and ripening of Vitis vinifera L. grape varieties in São Joaquim, southern Brazil: a new South American wine growing region. Ciencia e Investigación Agraria 37:61-75.

GRIS EF et al. 2011. Proanthocyanidin profile and antioxidant capacity of Brazilian Vitis vinifera red wines. Food Chemistry 126:213-220.

JACKSON DI \& LOMBARD PB. 1993. Environmental and management practices affecting grape composition and wine quality - a review. American Journal of Enology and Viticulture 44:409-430.

JONES G et al. 2004. Geology and wine. Modeling viticultural landscapes: A GIS Analysis of the Terroir Potential in the Umpqua Valley of Oregon. Geoscience 31:167-178.

KLIEWER WM. 1970. Effect of day temperature and light intensity on coloration of Vitis vinifera L. Grapes. Journal of the American Society for Horticultural Science 95:693697.

KLIEWER WM \& TORRES R. 1972. Effect of controlled day and night temperatures on grape coloration. American Journal of Enology and Viticulture 23:71-77.

KURTURAL SK et al. 2006. Utilizing GIS Technologies in Selection of Suitable Vineyard Sites. International Journal of Fruit Scienc 6:87-107.

MALINOVSKI LI et al. 2012. Highlands of Santa Catarina/ Brazil: a region with high potential for wine production. Acta Horticulturae 931:433-440.

MANDELLI F. 2006. Comportamento meteorológico e sua influência na vindima de 2006 na Serra Gaúcha. Passo Fundo: Embrapa. 4p. (Comunicado Técnico, 67).

MASSIGNAN AM \& PANDOLFO C. 2006. Estimativa das médias das temperaturas máximas, médias e mínimas do ar decendiais e anuais do estado de Santa Catarina. Florianópolis: Epagri. 26p. (Documentos 224).

MELLO LMR \& MACHADO CEI. 2013. Cadastro vitícola do Rio Grande do Sul: 2008 a 2012. Brasília: Embrapa. CD-Rom.

PANDOLFO C et al. 2007. Atlas climatológico digital do Estado de Santa Catarina. Florianópolis: Epagri. CD-Rom. PROTAS JFS. 2012. A marca coletiva como estratégia organizacional: o caso dos vinhos finos de altitude de Santa Catarina. Bento Gonçalves: Embrapa Uva e Vinho, Bento Gonçalves, 19p. (Documentos 77)

REGINA MA et al. 2006. Novos pólos vitícolas para produção de vinhos finos em Minas Gerais. Informe Agropecuário 27:234:111-118.

REGINA MA et al. 2006b. Implantação e manejo do vinhedo para produção de vinhos de qualidade. Informe Agropecuário 27:16-31.

REGINA M A et al. 2010. Influência da altitude na qualidade das uvas Charonnay e Pinot Noir em Minas Gerais. Revista Brasileira de Fruticultura 32:143-150.

REYNOLDS AG et al. 1986. Influence of cluster exposure on fruit composition and wine quality of Seyval blanc grapes. Vitis 25:85-95.

ROSIER JP. 2006. Vinhos de altitude: característica e potencial na produção de vinhos finos brasileiros. Informe Agropecuário 27:105-110.

SANTOS HP. 2006. Aspectos ecofisiográficos na condução da videira e sua influência na produtividade do vinhedo e na qualidade dos vinhos. Embrapa Uva e Vinhos: Bento Gonçalves. 9 p. (Comunicado Técnico 71).

SARMENTO EC et al. 2008. Sistema de informação geográfica como apoio ao levantamento detalhado de solos do Vale dos Vinhedos. Revista Brasileira de Ciência do Solo 32:2795-2803.

SCOTT ES et al. 2010. Fungal contaminants in the vineyard and wine quality. In: REYNOLDS AG. (ed). Managing wine quality. Volume1: Viticulture and wine quality. CRC Press: Boca Raton. p.481-514.

SDS 2013. Levantamento Aerofotogramétrico do Estado de Santa Catarina. Secretaria de Estado do Desenvolvimento Econômico Sustentável. Florianópolis. (Relatório de Gestão).

SENTELHAS PC. 1998. Aspectos climáticos para a viticultura tropical. Informe Agropecuário 194:9-14.

SMART RE et al. 1988. Light quality and quantity effects on fruit ripening for Cabernet Sauvignon. American Journal of Enology and Viticulture 39:250-258.

SPAYD S et al. 2002. Separation of sunlight and temperature effects on the composition of Vitis vinifera cv. Merlot berries. American Journal of Enology and Viticulture 53:171-182.

UBERTI AAA et al. 1992. Metodologia para classificação da aptidão de uso das terras do Estado de Santa Catarina. Florianópolis, SC: Epagri. 19p. (Documentos 119).

VAN LEEUWEN C \& SEGUIN G. 2006. The Concept of Terroir in Viticulture. Journal of Wine Research 17:1-10.

VAN LEEUWEN C. 2010. Terroir: the effect of the physical environment on vine growth, grape ripening and wine 
sensory attributes. In: REYNOLDS AG. (ed). Managing wine quality. Volume1: Viticulture and wine quality. CRC Press: Boca Raton. p.273-315.

VAUDOUR E. 2002. The quality of grapes and wine in relation to geography: Notions of terroir at various scales. Journal of Wine Research 13:117-141.

VIEIRA VF \& ZAMPARETTI AF. 2010. Um método para o georreferenciamento dos vinhedos. Mundo Geo.

WOLF TK \& BOYER JD. 2003. Vineyard Site Selection. Virginia Cooperative Extension. 36p. 